May 20, 2022 1:25 WSPC/INSTRUCTION FILE ws-ijmpd

International Journal of Modern Physics D

(C) World Scientific Publishing Company

\title{
Compact Stars in Hadron and Quark-Hadron Models
}

\author{
Stefan Schramm \\ FIAS, Goethe University, Ruth Moufang Str. 1 \\ 60438 Frankfurt, Germany \\ schramm@th.physik.uni-frankfurt.de \\ Verônica Antocheviz Dexheimer \\ Department of Physics, Gettysburg College, 300 North Washington Street \\ Gettysburg, PA 17325, USA \\ Received Day Month Year \\ Revised Day Month Year \\ Communicated by Managing Editor
}

\begin{abstract}
We investigate strongly interacting dense matter and neutron stars using a flavor-SU(3) approach based on a non-linear realization of chiral symmetry as well as a hadronic flavor$\mathrm{SU}(2)$ parity-doublet model. We study chiral symmetry restoration and the equation of state of stellar matter and determine neutron star properties using different sets of degrees of freedom. Finally, we include quarks in the model approach. We show the resulting phase diagram as well as hybrid star solutions for this model.
\end{abstract}

Keywords: chiral symmetry; neutron star; quark star.

\section{Introduction}

The study of strongly interacting matter at extreme conditions of temperature and density is at the forefront of modern nuclear physics. This regime covers the physics of ultrarelativistic heavy-ion collisions as well as important aspects of nuclear astrophysics. Whereas the determination of the phase structure of excited strongly interacting matter at high temperature and baryon densities, as pursued in heavyion collisions, involves relatively high temperatures, the complementary study of the structure of compact stars is directly related to the properties of very dense and rather cold matter.

In order to study all these regimes in a unified model approach we developed an effective chiral $\mathrm{SU}(3)$ model that can be studied over the whole relevant range of chemical potentials and temperatures. Reflecting the correct degrees of freedom of the system after the transition to a quark-gluon plasma state we include quarks and the Polyakov loop as order parameter for the deconfinement phase transition. 


\section{The Hadronic Model}

The model used in our calculations is an extended $\sigma-\omega$ chiral model, based on a nonlinear realization of chiral symmetry, that includes the lowest $\mathrm{SU}(3)$ multiplets of baryons and mesons as well as an effective field that mimics the QCD scale anomaly. The expectation values of the scalar isoscalar fields correspond to the non-strange isoscalar $(\sigma$ field), isovector $(\delta)$ and strange $(s \bar{s})$ chiral quark condensates $(\zeta)$.

The interactions between baryons and the scalar and vector mesons are linear and read (assuming static systems, including only the time component of the vector fields)

$$
\begin{gathered}
\mathcal{L}_{\mathrm{BM}}=-\sum_{i} \bar{\psi}_{i}\left(g_{i \sigma} \sigma+g_{i \delta} \delta+g_{i \zeta} \zeta\right) \psi_{i} \\
\mathcal{L}_{\mathrm{BV}}=-\sum_{i} \bar{\psi}_{i}\left(g_{i \omega} \gamma_{0} \omega^{0}+g_{i \rho} \gamma_{0} \rho^{0}+g_{i \phi} \gamma_{0} \phi^{0}\right) \psi_{i},
\end{gathered}
$$

where $\omega, \rho$, and $\phi$ are the non-strange isoscalar, isovector and strange vector fields, respectively. The index $i$ sums over the baryon octet $(N, \Lambda, \Sigma, \Xi)$. Additional terms include mass terms and quartic self-interactions of the vector mesons, whereas the self-interactions of the scalar mesons induce the spontaneous breaking of chiral symmetry. The effect of non-zero current quark masses is modelled by introducing an explicit chiral-symmetry breaking term (for more details on the full Lagrangian see 1 ). The vacuum masses of the baryons are generated through their coupling to the chiral condensates.

For the baryon-vector couplings $g_{i \omega}$ and $g_{i \phi}$ pure $f$-type coupling is assumed as discussed in 1, $g_{i \omega}=\left(n_{q}^{i}-n_{\bar{q}}^{i}\right) g_{8}^{V}, g_{i \phi}=-\left(n_{s}^{i}-n_{\bar{s}}^{i}\right) \sqrt{2} g_{8}^{V}$, where $g_{8}^{V}$ denotes the vector coupling of the baryon octet and $n^{i}$ the number of constituent quarks of species $i$ in a given hadron. The resulting relative couplings are in accordance with additive quark model constraints, coupling only the $\phi$ vector mesons to strange baryons.

All parameters of the model are fixed by either symmetry relations, hadronic vacuum observables or nuclear matter saturation properties (see 1 ). In addition, the model also provides a satisfactory description of realistic (finite-size and isospin asymmetric) nuclei and neutron stars 112. Using this approach the equations of motion are solved for isospin symmetric nuclear matter as well as star matter by including leptons and requiring charge neutrality. Fig. 1 shows the resulting equation of state of cold symmetric nuclear and star matter. The parameters were slightly tuned from the fit values used in calculations of finite nuclei 3 to explore the range of possible maximum star masses, while retaining a reasonable description of saturated nuclear matter. The nuclear compressibility has a still acceptable value of $297 \mathrm{MeV} 2$. The results for three choices of baryonic degrees of freedom are shown, which comprise (a) nucleons, (b) nucleons and hyperons, and (c) nucleons, hyperons and spin $3 / 2$ baryonic resonances. As expected, with increasing number of particle species the equation of state becomes softer at larger densities. Using these equa- 
tions of state in a calculation of the properties of a spherical and static neutron star, integrating the Tolman-Oppenheimer-Volkoff (TOV) equations 2, we obtain maximum star masses between 1.93 and 2.12 solar masses depending on the number of degrees of freedom taken into account (Fig. 2). One can observe that even including $\Delta$ baryons in the calculation rather large star masses with typical radii of about $12 \mathrm{~km}$ are possible. Looking into the corresponding particle densities, shown in Fig. 3, the hyperon content of the star is relatively small with $\Lambda$ baryons appearing at about 3 times nuclear matter groundstate density and the $\Sigma^{-}$at $4 \rho_{0}$, respectively. The latter result is due to the fact that in the model the $\Sigma$ potential in nuclear matter is positive $\left(U_{\Sigma}=5.35 \mathrm{MeV}\right)$. In case one includes the baryonic spin $3 / 2$ decuplet, that is $\Delta$ baryons for the densities occurring in compact stars, the particle mix looks very different as can be seen in Fig. 4 . The $\Delta^{-}$is populated

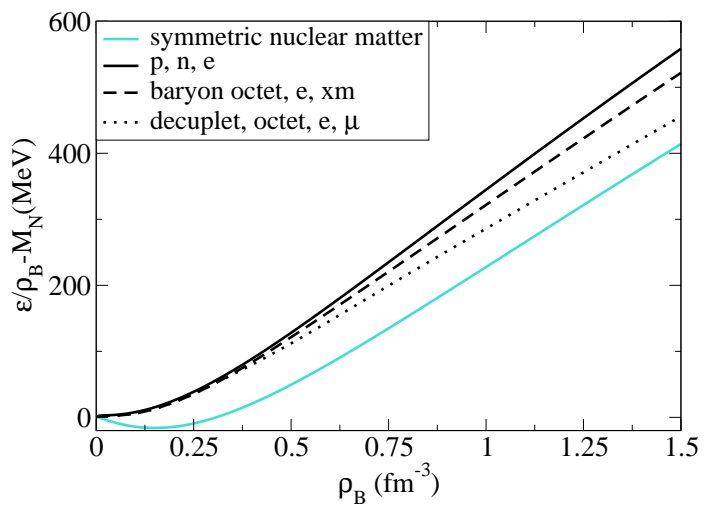

Fig. 1. Equation of State of isospin-symmetric nuclear matter and stellar matter including nucleons, nucleons and hyperons, and in addition spin3/2 baryons.



Fig. 2. Mass-Radius diagram for neutron star solutions using different EOS as shown in Fig. 1. 
above $2 \rho_{0}$ and the hyperons are shifted to larger densities. Note, however, that the results depend strongly on the largely unknown value of the vector coupling of the $\Delta$ to the $\omega$ meson, here taken to be identical to the nucleon- $\omega$ coupling.

One possible effect that might occur in neutron stars is meson, in particular $K^{-}$, condensation 4 . Using our model, in a calculation of the critical densities for the onset of the condensation in neutron and proto-neutron stars we find that for cold stars the condensate sets in at values of about 6 times nuclear matter saturation density whereas for proto-neutron stars the value is even higher. These values are beyond the maximum central densities inside of the stars such that kaon condensation does not affect stellar properties 516 .

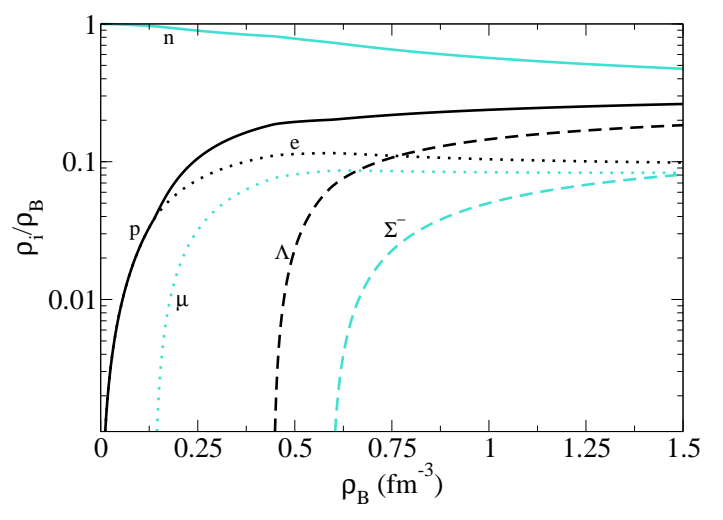

Fig. 3. Particle abundances as function of density for a star including nucleons and hyperons.

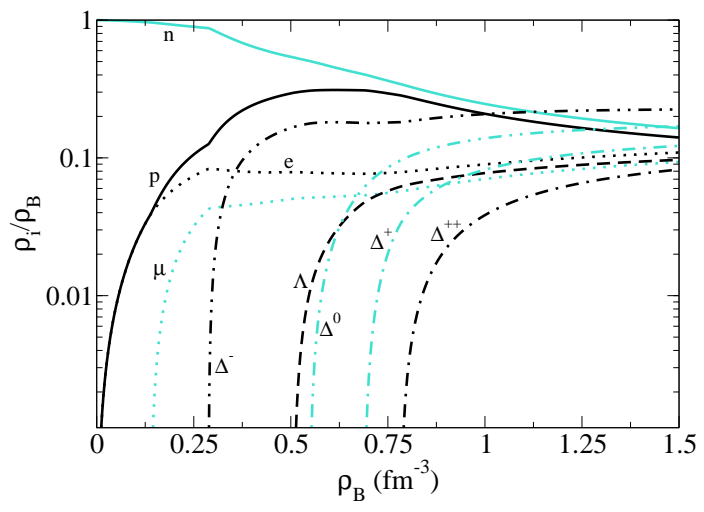

Fig. 4. Particle abundances as function of density for a star including nucleons, hyperons, and $\Delta$ resonances. 


\section{The Parity-Doublet Model}

Using an alternative approach of formulating a chirally symmetric Lagrangian we consider the parity doublet model originally discussed in 7]. Here, one adopts the so-called "mirror assignment" for the positive and negative parity nucleon states ( $N_{+}$and $N_{-}$, we restrict ourselves to $\mathrm{SU}(2)$, in this case), grouping both states in the same multiplet. Under left- and right-handed transformations $S U_{L}(2) \times S U(2)_{R}$ transformations the two nucleon fields $\psi_{1}$ and $\psi_{2}$ transform non-trivially as:

$$
\begin{array}{ll}
\psi_{1 R} \longrightarrow R \psi_{1 R}, & \psi_{1 L} \longrightarrow L \psi_{1 L} \\
\psi_{2 R} \longrightarrow L \psi_{2 R}, & \psi_{2 L} \longrightarrow R \psi_{2 L} .
\end{array}
$$

In a similar way as $\sigma$ and $\pi$ are parity partners in the linear realization of chiral symmetry this allows for a chirally invariant nucleonic mass term in the Lagrangian:

$$
m_{0}\left(\bar{\psi}_{2} \gamma_{5} \psi_{1}-\bar{\psi}_{1} \gamma_{5} \psi_{2}\right)=m_{0}\left(\bar{\psi}_{2 L} \psi_{1 R}-\bar{\psi}_{2 R} \psi_{1 L}-\bar{\psi}_{1 L} \psi_{2 R}+\bar{\psi}_{1 R} \psi_{2 L}\right),
$$

where $m_{0}$ represents a bare mass parameter. After diagonalizing the quadratic terms in the Lagrangian one obtains the unmixed fields, $N_{+}$and $N_{-}$, that are the standard nucleon and its parity partner. In the limit of chiral symmetry restoration $(\sigma=0)$ both nucleonic states are degenerate, but attain a finite mass $m_{0}$. This doubling of degenerate nucleonic states is the characteristic feature of this type of chiral model.

Using the parity-doublet ansatz combined with a linear sigma model including the $\omega$ meson (for achieving the correct nuclear matter saturation properties) and the $\rho$ meson (for reproducing the phenomenological value for the asymmetry energy of $32 \mathrm{MeV}$ ) one can determine the corresponding field values and the equation of state by solving the equations of motion of the model in mean-field approximation 8 . In Fig. 5 the resulting $\sigma$ field, the scalar condensate, is shown. The field becomes smaller with increasing density tending towards chiral symmetry restoration. The structure in the curve for star matter at about 2 times $\rho_{0}$ corresponds to the onset of populating the parity partner of the neutron. This can directly been seen in Fig. 6. Above $4 \rho_{0}$ the parity partner of the proton shows up.

Inserting the equation of state into the star calculation one obtains a maximum star mass of 1.85 solar masses. Including sizeable vector meson self-interaction terms leads to very low star masses in contradiction to values of observed stars. Going beyond the mean-field approximation we repeated the calculations using the Relativistic Hartree Approximation (RHA), which in general generates comparable results slightly lowering the nuclear matter compressibility 9 .

In general, for the parity partners to be populated in the interior of the star a relatively low vacuum mass of the $N^{-}$baryon below $1380 \mathrm{MeV}$ is required. In addition, in order to maintain reasonably small values for the compressibility one has to assume a high value of the mass parameter $m_{0}>800 \mathrm{MeV}$. A full analysis of the question whether with these values one can still be in accordance with lowenergy hadronic data like pion-nucleon scattering is still an open question 9 . 


\section{Including Quarks}

Lattice QCD calculations show that the phase transition from hadrons to quarks and gluons is a smooth crossover at vanishing chemical potential. Some lattice calculations at finite chemical potential $\mu_{B}$, which are still notoriously difficult to perform, suggest a critical end-point of a line of first-order phase transitions in the T- $\mu_{B}$ plane 10 . Calculations that connect hadrons and quarks in two different model approaches are not able to reproduce such a transition behavior. Therefore we extend our hadronic model by including quarks and an effective field $\Phi$ for the Polyakov loop, following 11/1213, in order to describe the deconfinement phase transition in a single unified approach 14 . The potential for the Polyakov loop reads:

$$
U=\left(a_{0} T^{4}+a_{1} \mu_{B}^{4}+a_{2} T^{2} \mu_{B}^{2}\right) \Phi^{2}+a_{3} T_{0}^{4} \ln \left(1-6 \Phi^{2}+8 \Phi^{3}-3 \Phi^{4}\right) .
$$

The parameters are obtained by fitting the values to lattice QCD results at vanishing chemical potential. The $\mu_{B}$-dependent terms are fixed by reproducing the critical end-point as computed in 10. The switch between quark and baryon degrees of freedom is achieved in an effective wav, shifting the baryon/quark masses

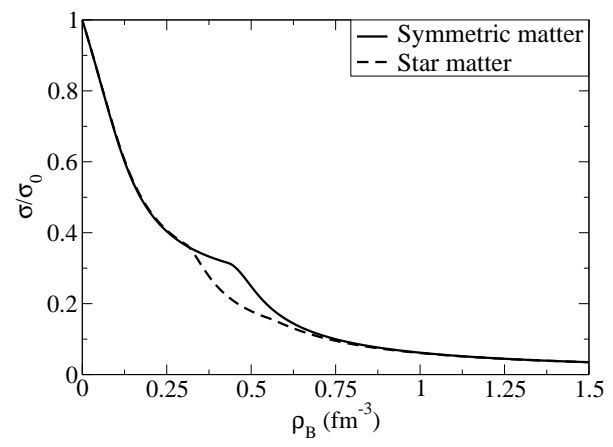

Fig. 5. Scalar condensate as function of density in the parity-doublet model. The mass for the parity partner $N_{-}$is set to $1200 \mathrm{MeV}$.

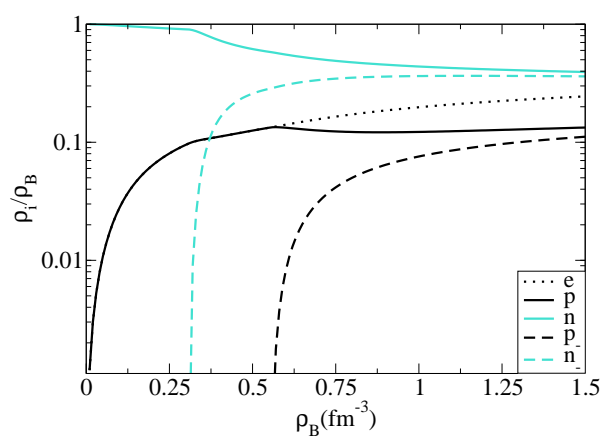

Fig. 6. Densities of particle species as function of density in the parity-doublet model. 
to high/small values for small/high Polyakov loop values using a simple additional mass term for quarks and baryons, such that their effective masses read:

$$
\begin{gathered}
m_{b}^{*}=g_{b \sigma} \sigma+g_{b \delta} \tau_{3} \delta+g_{b \zeta} \zeta+m_{0 b}+g_{b \Phi} \Phi^{2}, \\
m_{q}^{*}=g_{q \sigma} \sigma+g_{q \delta} \tau_{3} \delta+g_{q \zeta} \zeta+m_{0 q}+g_{q \Phi}(1-\Phi),
\end{gathered}
$$

where $m_{0}$ are small explicit mass terms and $g_{b \Phi}$ and $g_{q \Phi}$ are the coupling constants of the Polyakov loop to baryons and quarks, respectively 14 . Using this approach we obtain the phase diagram shown in Fig. 7. Note that we also obtain a realistic first-order liquid-gas phase transition. The transition to quarks at zero temperature occurs at $4 \rho_{0}$.

It is straightforward to calculate star masses in this model. The result in Fig. 8 shows a maximum star mass of 1.92 solar masses. Using a Maxwell construction, assuming local charge neutrality of the star matter, the family of stable stars ends with the appearance of quarks and a significant softening of the equation of state. Assuming global charge neutrality one obtains a mixed phase of quarks and hadrons in the inner $2 \mathrm{~km}$ core of the maximum mass star.

\section{References}

\section{References}

1. P. Papazoglou, D. Zschiesche, S. Schramm, J. Schaffner-Bielich, H. Stöcker and W. Greiner, Phys. Rev. C 59, 411 (1999).

2. V. Dexheimer and S. Schramm, Astrophys. J. 683, 943 (2008).

3. S. Schramm, Phys. Rev. C66 064310 (2002).

4. D. B. Kaplan and A. E. Nelson, Phys. Lett. B 175, 57 (1986); A. E. Nelson and D. B. Kaplan, ibid, 192, 193 (1987).

5. A. Mishra, A. Kumar, S. Sanyal, S. Schramm, Eur.Phys.J.A41:205-213 (2009).

6. A. Mishra, A. Kumar, S. Sanyal, V. Dexheimer, submitted to Phys. Rev. C., arxiv 0905.3518 [nucl-th].

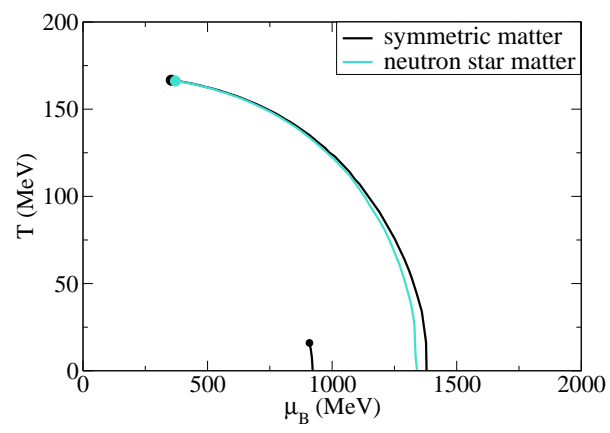

Fig. 7. Phase diagram in $\mu_{B}$ and $T$ for the Hadron-Quark model. First-order transition lines for the chiral and deconfinement transitions as well as for the liquid-gas phase transitions are shown as calculated in the model. 
7. C. DeTar, T. Kunihiro, Phys. Rev. D 39, 2805 (1989).

8. V. Dexheimer, S. Schramm, and D. Zschiesche, Phys. Rev. C 77, 025803 (2008).

9. V. Dexheimer, G. Pagliara, L. Tolos, J. Schaffner-Bielich, S. Schramm, Eur. Phys. J. A 38, 105-113 (2008)

10. Z. Fodor and S. D. Katz, JHEP 0404 (2004) 050.

11. K. Fukushima, Phys. Lett. B 591, 277 (2004) arXiv:hep-ph/0310121.

12. C. Ratti, M. A. Thaler and W. Weise, Phys. Rev. D 73, 014019 (2006)

13. S. Roessner, C. Ratti and W. Weise, Phys. Rev. D 75, 034007 (2007)

14. V. Dexheimer and S. Schramm, , preprint arXiv:0901.1748 [astro-ph], submitted to Phys Rev C; V. Dexheimer and S. Schramm, Nucl. Phys. A827, 988 (2009).

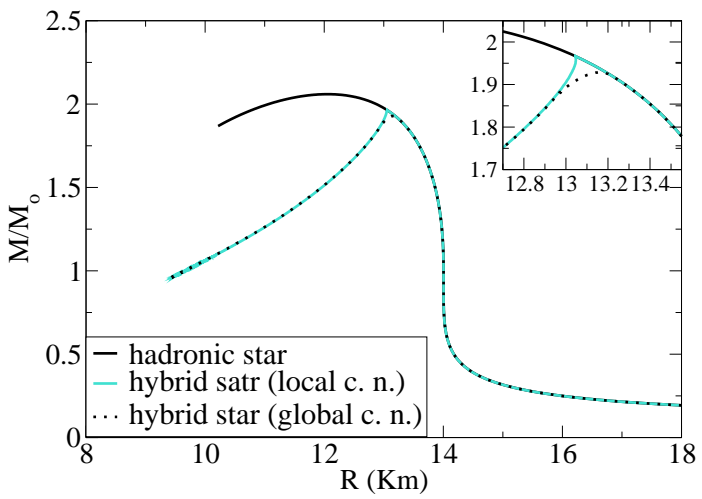

Fig. 8. Mass-radius relation of compact stars in the Hadron-Quark model. The inset shows the different results for a Maxwell and Gibbs construction of the phase transition. The Gibbs construction generates a mixed hadron-quark phase that smooths the sharp transition. 